\title{
Extranodal NK/T-Cell Lymphoma Occurring Primarily in the Eyes and with Central Nervous System Relapse
}

\section{Chong Wei 1 \\ Lu Zhang \\ Wei Zhang \\ Daobin Zhou}

Department of Hematology, Peking Union Medical College Hospital, Chinese Academy of Medical Sciences \& Peking Union Medical College, Beijing, People's Republic of China
Correspondence: Daobin Zhou

Email zhoudb@pumch.cn

\begin{abstract}
A 56-year-old man complaining of blurred vision was referred to the ophthalmologist. He was initially diagnosed with uveitis and showed no response to steroid treatment. Diagnostic vitrectomy and skin lesion biopsy performed later confirmed the diagnosis of extranodal NK/T cell lymphoma (ENKTL), nasal type. Despite sufficient systemic chemotherapy, the patient developed central nervous system (CNS) relapse 3 months after achieving systemic remission. The diagnosis and treatment of this patient required multidisciplinary teamwork between ophthalmologists, pathologists, and hematologists. This report focused on the diagnostic planning and optimal treatment strategy for this patient.
\end{abstract}

Keywords: extranodal NK/T-cell lymphoma, intraocular lymphoma, chemotherapy, central nervous system

\section{Introduction}

The extranodal natural killer/T-cell lymphoma, nasal type (ENKTL) is a relatively rare and highly aggressive tumor, comprising less than $1.5 \%$ of all non-Hodgkin's lymphomas worldwide. The intraocular involvement of ENKTL, especially as the first sign of disease onset, is a rare presentation of this uncommon disease. Its diagnosis remains challenging due to its rarity and ability to masquerade as noninfectious or infectious uveitis.

We report a case of ENKTL with ocular presentation as the initial manifestation, with CNS relapse within a short time after achieving systemic complete remission. This report highlights the need for clinical suspicion of intraocular lymphoma (IOL) in cases of severe and sudden bilateral uveitis that does not respond to steroid therapy and the importance of diagnostic vitrectomy. Not only is the diagnosis of this patient challenging, but also the treatment process. This report also focused on the optimal treatment strategy for patients with ENKTL with intraocular involvement.

\section{Case Report}

A 56-year-old Chinese man presented to the ophthalmic clinic complaining of blurred vision and redness in both eyes that has been present for a month. On inspection, there was bilateral conjunctival chemosis, anterior chamber flare, and a hazy vitreous. The fundus could not be viewed clearly, but small patches of yellow-white lesions could be faintly seen (Figure 1A and B). Fundus fluorescein 

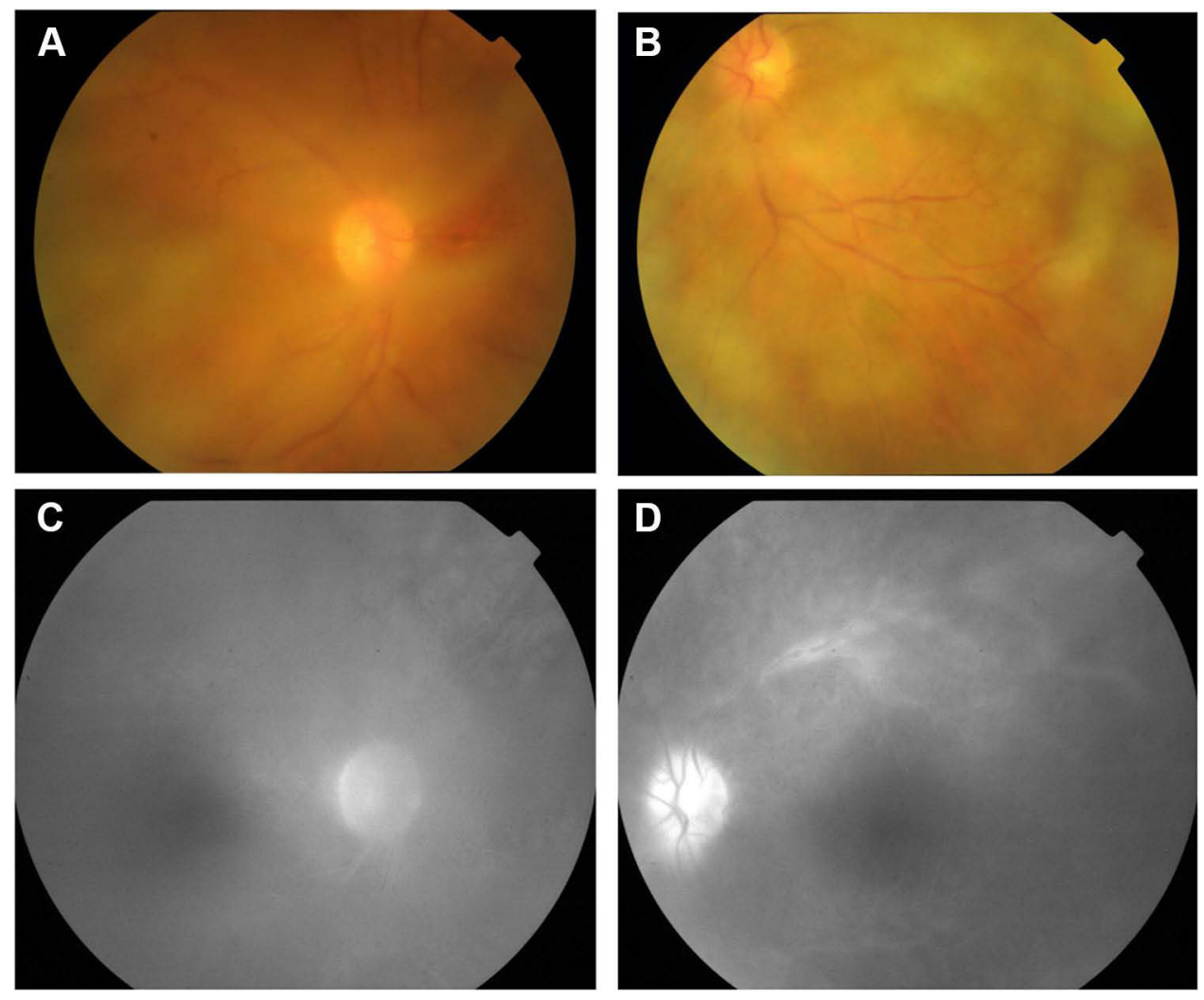

Figure I Fundus examination is not clear, and small patches of yellow-white lesions could be seen. (A) OD; (B) OS. Fundus fluorescein angiography shows retinal venous engorgement with fluorescein leakage and high fluorescence of the optic disc. (C) OD; (D) OS.

angiography revealed retinal venous engorgement with fluorescein leakage and hyperfluorescence on the optic disc (Figure 1C and D). Based on these clinical manifestations, the patient was diagnosed with bilateral uveitis and was started on a systemic methylprednisolone treatment. After transient improvement, his visual acuity rapidly deteriorated. In addition, the patient complained of fever with an evening peak and night sweats. Subcutaneous nodules with skin ulceration were observed on the trunk and upper extremities. IOL was suspected, and a diagnostic vitrectomy of the left eye was performed. Pathological examination of the vitreous humor revealed infiltration of atypical lymphoid cells. Flow cytometry of the vitreous humor confirmed the NK cell immunophenotype, which was positive for CD56 and CD38 and negative for CD3. The interleukin-6 and interleukin-10 levels of the vitreous humor were $299.5 \mathrm{pg} / \mathrm{mL}$ and $27.2 \mathrm{pg} / \mathrm{mL}$ with a ratio of 0.09 . Biopsies of the skin and subcutaneous nodules were also performed, and the histopathological findings and immunohistochemical staining were consistent with those of ENKTL (Figure 2). In situ hybridization for Epstein-Barr virus-encoded RNA (EBER) was also positive. Tests for T-cell antigen receptor gene rearrangements were negative. The patient was transferred to the hematological department for further evaluation and treatment after the final diagnosis of ENKTL was made.

Blood tests, including complete blood count and liver and renal function, were normal. Epstein-Barr virus-DNA was highly positive $\left(67 \times 10^{4}\right.$ copies $\left./ \mathrm{mL}\right)$. Bone marrow biopsy, cerebrospinal fluid analysis, and magnetic resonance imaging of the brain showed no abnormalities. Positron emission tomography-computed tomography showed significant ${ }^{18} \mathrm{~F}$-fluorodeoxyglucose uptake in the skin, nasopharynx, small intestine, and kidneys (Figure 3). As such, he was diagnosed with stage IV ENKTL according to the Ann Arbor staging system. The patient was initially treated with six cycles of the SMILE regimen (dexamethasone, methotrexate, ifosfamide, L-asparaginase, and etoposide). A mid-treatment positron emission tomography-computed tomography scan after 4 cycles of chemotherapy demonstrated complete remission.

However, the patient developed drowsiness and dyspnea with acute onset and rapid progression while autologous stem-cell transplantation was being planned as consolidation therapy. Mechanical ventilation was initiated after the patient 

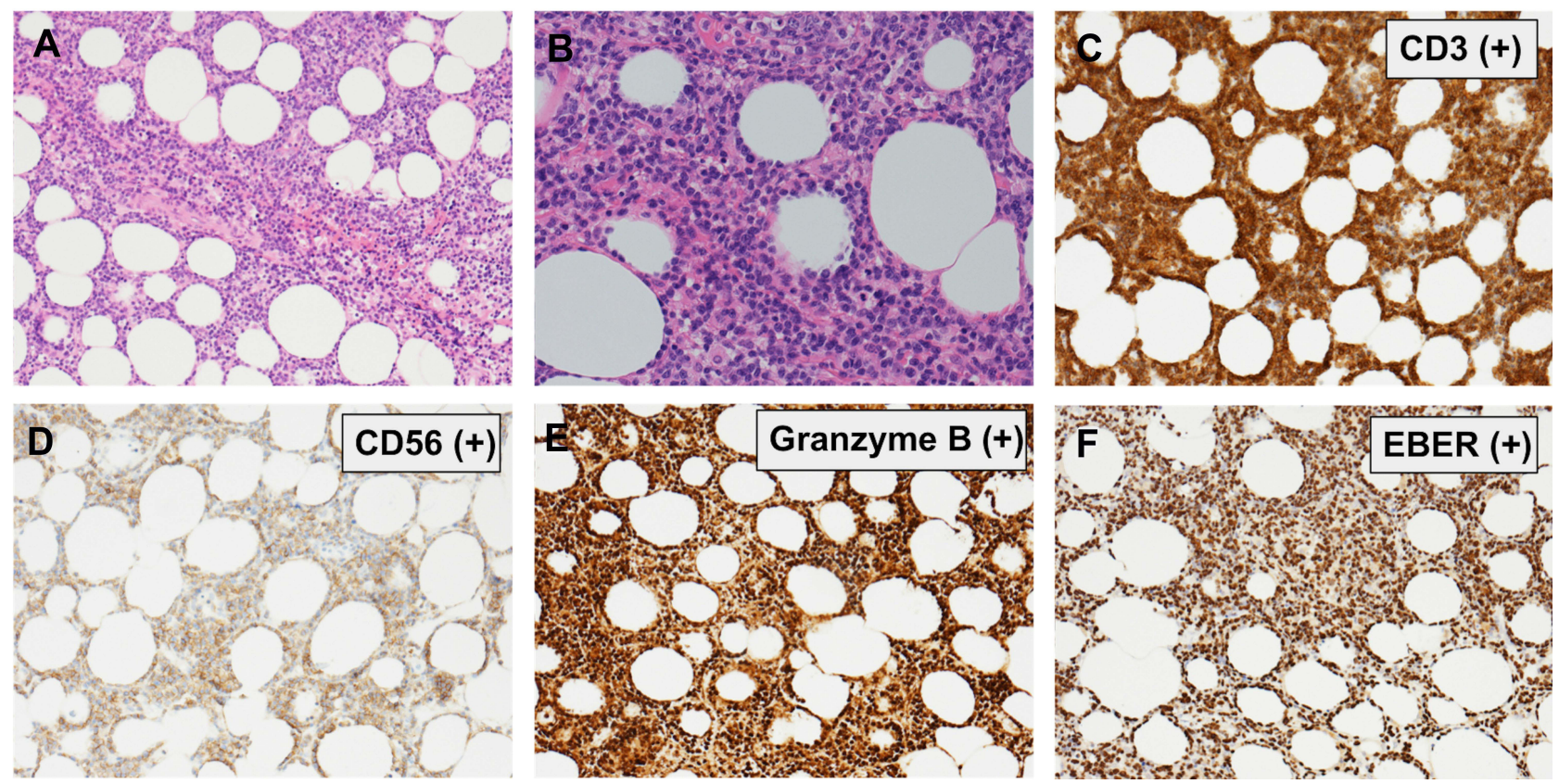

Figure 2 Histological and immunohistochemical staining of the tumor cells. (A and B) Monomorphic medium-sized lymphoid cells with angiocentric pattern (hematoxylin and eosin stain; original magnification $\times 100[A]$ and $\times 200[B]$ ); Immunostaining for CD3 (C), CD56 (D), granzyme B (E), and EBER by in situ hybridization (F) (original magnifications $\times 100)$.
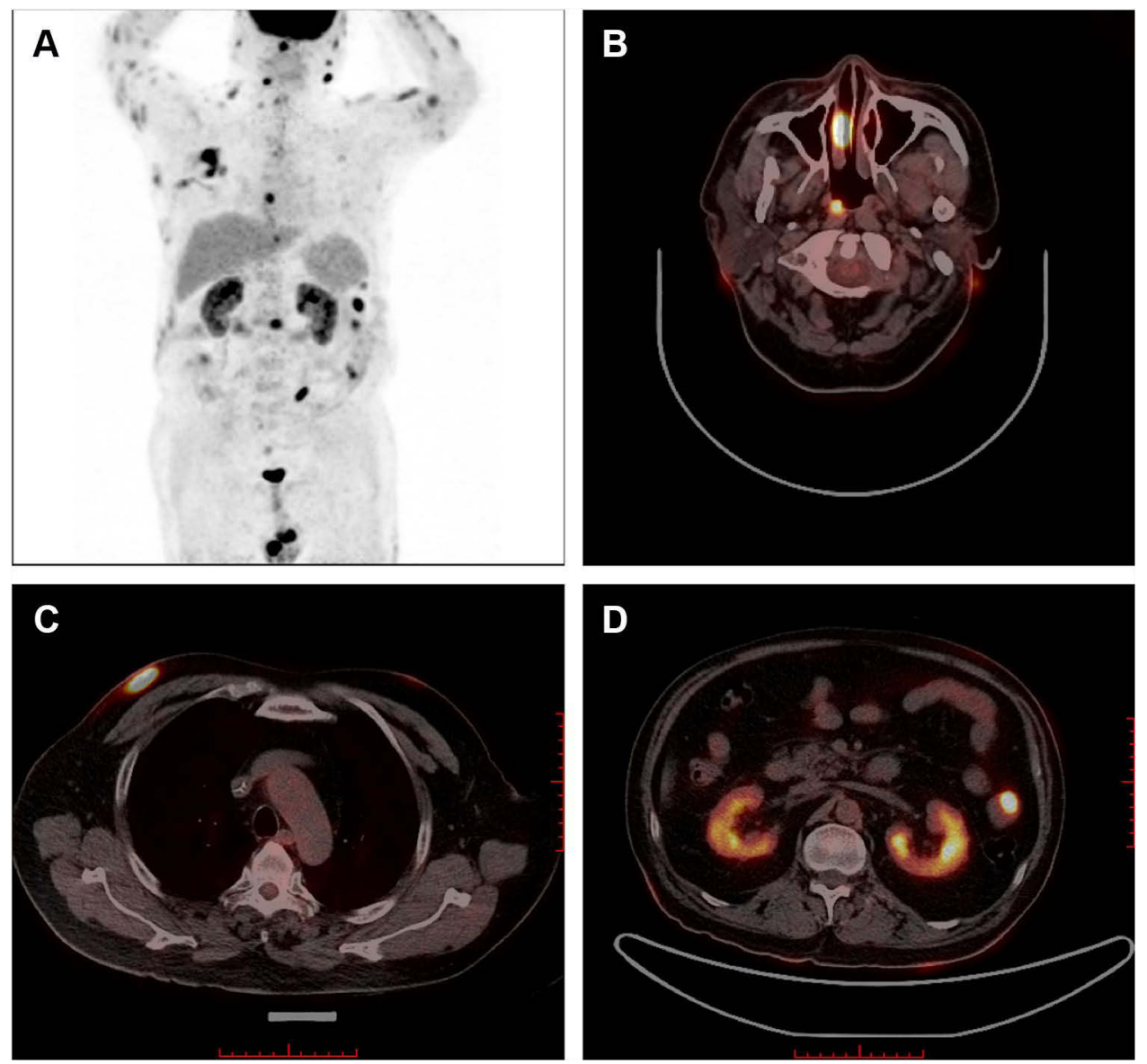

Figure 3 Positron emission tomography/computed tomography shows significant I8F-FDG uptake in multiple sites of the body (A), including the nasopharynx (B), skin and subcutaneous tissue of the right chest (C), small intestine, and kidneys (D). 
was transferred to the intensive care unit. Subsequent cerebrospinal fluid flow cytometry analysis revealed the presence of neoplastic NK cells. Magnetic resonance imaging of the brain showed no abnormalities. Based on the above results, the patient was diagnosed with CNS relapse with leptomeningeal involvement. He was treated with two cycles of antiprogrammed cell death 1 antibody (sintilimab) combined with P-GEMOX regimen (gemcitabine, oxaliplatin, and pegaspargase) as salvage treatment. A total of five cycles of intrathecal injection (10 mg methotrexate, $50 \mathrm{mg}$ cytarabine, and $5 \mathrm{mg}$ dexamethasone) were administered twice a week. His mental status gradually returned to normal, and dyspnea was relieved. However, the treatment was interrupted during the COVID-19 epidemic in China. He subsequently developed pancytopenia. Biopsy of the bone marrow showed infiltration of lymphoma cells. He received palliative treatment at a local hospital. The patient died of disease progression 12 months after the initial diagnosis and 3.5 months after the CNS relapse.

\section{Discussion}

The ENKTL is a rare and highly aggressive tumor that is associated with Epstein-Barr virus infection. It is more common in Asia and Latin America than in Western countries. ${ }^{1}$ ENKTL most often originates in the upper aerodigestive tract. However, ENKTL can have an extranasal presentation, with the skin, testis, and gastrointestinal tract being the most common sites of involvement. In an analysis of 136 ENKTL patients from the International NK/T-cell Lymphoma Project, $68 \%$ were of the nasal type, and only $26 \%$ were of the extranasal type. ${ }^{2}$ The extranasal type was reported as a negative prognostic factor in the prognostic index of natural killer lymphoma (PINK), as proposed by Kim et al. ${ }^{3}$

IOL is rare, accounting for less than $2 \%$ of ocular malignant. ${ }^{4}$ Most cases of IOL are of B-cell origin. However, there are also a few cases with NK/T-cell origin, with most representing a secondary manifestation. ${ }^{5}$ Few cases of intraocular NK/T-cell lymphoma have been reported in the literature. The largest case series was reported by Hon et al, who reviewed six ENKTL patients with ocular complications in Hong Kong. ${ }^{6}$ The patients in this study were divided into two distinct categories: uveitis/ vitritis (3 patients) and orbital infiltration (3 patients). Among the three patients with uveitis/vitritis, ocular symptoms preceded the diagnosis of lymphoma in two patients.

Abe et al reported a case of anterior uveitis diagnosed with rapidly progressive vision loss. ${ }^{7}$ The patient soon developed pericardial effusion and died from cardiac tamponade. The diagnosis of ENKTL was confirmed by necropsy. Meanwhile, Zhang et al reported a case of bilateral uveitis. ${ }^{8}$ Further examination revealed a neoplasm in the nasopharynx. A final diagnosis of ENKTL was made based on the pathology of the nasopharyngeal neoplasm. Unfortunately, this patient died of multiple organ failure. Similarly, in our case, although the patient was also first misdiagnosed with uveitis, the diagnostic vitrectomy was performed in time, allowing the patient to receive systemic chemotherapy. Clinicians should always be cautious of IOL in cases of bilateral sudden and severe uveitis that do not respond to steroid therapy or recur. Patients with suspected IOL should undergo a cytopathologic examination of the vitreous fluid or vitrectomy to ensure a timely diagnosis, which is essential for improving prognosis.

Due to the rarity of ENKTL, standard and optimal treatments have not been established. In recent years, the prognosis of ENKTL patients has improved due to the use of chemoradiotherapy and L-asparaginase-based regimens, such as SMILE $^{9}$ and P-GEMOX. ${ }^{11}$ However, few studies have focused on the management of ENKTL with intraocular involvement. An ocular manifestation is a unique presentation of lymphoma that may warrant additional treatment. First, due to the blood-ocular barrier, systemic chemotherapy is not sufficient to achieve a therapeutic concentration in the eyes. ${ }^{11}$ Systemic chemotherapy combined with eye-dedicated therapy, including intravitreal methotrexate injection or ocular radiation, may be an effective way to achieve both local and systemic control of the disease. ${ }^{12}$ Second, intraocular involvement is associated with a high incidence of CNS relapse in patients with primary CNS lymphoma. ${ }^{13}$ However, the risk factors for CNS relapse in ENKTL are poorly defined. Recently, Kim et al proposed a central nervous system-prognostic index of natural killer (CNS-PINK) model to predict CNS relapse in patients with ENKTL. ${ }^{14}$ This model included the intermediate/high prognostic index of natural killer (PINK) score and extranodal involvement $\geq 2$ as predictors of CNS relapse, with 1 point for each factor. ${ }^{3,14}$ Patients with these two factors were at high risk of CNS relapse, with a 2-year CNS risk of $22.8 \%$. The PINK score of this patient was 2 (high risk) with $>2$ extranodal involvement, which is stratified as having a high risk of CNS relapse in this model. Intrathecal treatment and high-dose systemic methotrexate are commonly used in the treatment of primary CNS lymphoma and DLBCL with CNS involvement. This patient was treated with SMILE chemotherapy, which also included methotrexate. However, the dose of methotrexate in the SMILE regimen is $2 \mathrm{~g} / \mathrm{m}^{2}$, which is not high dose. The insufficient dose of systemic methotrexate and lack of 
intrathecal or intravitreal treatment might have been related to the CNS relapse in this patient. Adding an intravitreal treatment and high-dose methotrexate to systemic chemotherapy might promote longer progression-free survival in this patient. To our knowledge, this was the first case of ENKTL with ocular presentation as the initial manifestation of CNS relapse.

\section{Conclusion}

This case report highlighted the need for clinical suspicion of IOL in cases of bilateral sudden and severe uveitis that do not respond to steroid therapy. Patients with suspected IOL should undergo a cytopathologic examination of the vitreous fluid or vitrectomy to ensure a timely diagnosis. Systemic chemotherapy combined with eye-dedicated therapy, including intravitreal methotrexate injection or ocular radiation, may be an effective way to achieve both local and systemic control of the disease.

\section{Ethics and Consent}

The study was done in accordance with the Declaration of Helsinki.

The patient gave consent for the publication of identifiable details to be published in the journal of cancer management and research. Informed consent was obtained from his daughter after his death. Institutional approval was not required to publish the case details.

\section{Disclosure}

The authors declare that they have no conflicts of interest for this work.

\section{References}

1. Chan JKC, Quintanilla-Martinez L, Ferry JA, et al. Extranodal NK/ T-cell lymphoma, nasal type. In: Swerdlow SH, Campo E, Harris NL, et al. editors. WHO Classification of Tumours of Haematopoietic and Lymphoid Tissues. Lyon: International Agency for Research on Cancer; 2017:368-371.
2. Au WY, Weisenburger DD, Intragumtornchai T, et al. Clinical differences between nasal and extranasal natural killer/T-cell lymphoma: a study of 136 cases from the international peripheral T-cell lymphoma project. Blood. 2009;113:3931-3937. doi:10.1182/blood2008-10-185256

3. Kim SJ, Yoon DH, Jaccard A, et al. A prognostic index for natural killer cell lymphoma after non-anthracycline-based treatment: a multicentre, retrospective analysis. Lancet Oncol. 2016;17:389-400.

4. Reddy EK, Bhatia P, Evans RG. Primary orbital lymphomas. Int J Radiat Oncol Biol Phys. 1988;15:1239-1241. doi:10.1016/ 0360-3016(88)90210-6

5. Sagoo MS, Mehta H, Swampillai AJ, et al. Primary intraocular lymphoma. Surv Ophthalmol. 2014;59:503-516. doi:10.1016/j. survophthal.2013.12.001

6. Hon C, Kwok AK, Shek TW, et al. Vision-threatening complications of nasal T/NK lymphoma. Am J Ophthalmol. 2002;134:406-410. doi:10.1016/S0002-9394(02)01520-9

7. Abe RY, Pinto RD, Bonfitto JF, et al. Ocular masquerade syndrome associated with extranodal nasal natural killer/T-cell lymphoma: case report. Arq Bras Oftalmol. 2012;75:430-432. doi:10.1590/S000427492012000600013

8. Zhang F, Duan X, Liu K. A case report of an extranodal NK/T-cell lymphoma nasal type, occurring primarily in eyes with masquerade syndrome. Medicine (Baltimore). 2019;98:e14836. doi:10.1097/ MD.0000000000014836

9. Yamaguchi M, Kwong YL, Kim WS, et al. Phase II study of SMILE chemotherapy for newly diagnosed stage IV, relapsed, or refractory extranodal natural killer (NK)/T-cell lymphoma, nasal type: the NK-cell tumor study group study. $J$ Clin Oncol. 2011;29:4410-4416. doi:10.1200/JCO.2011.35.6287

10. Wang JH, Liang W, Liu CC, et al. Efficacy of combined gemcitabine, oxaliplatin and pegaspargase (P-gemox regimen) in patients with newly diagnosed advanced-stage or relapsed/refractory extranodal NK/T-cell lymphoma. Oncotarget. 2016;7:29092-29101. doi:10.18632/oncotarget. 8647

11. Tomi M, Hosoya K. The role of blood-ocular barrier transporters in retinal drug disposition: an overview. Expert Opin Drug Metab Toxicol. 2010;6:1111-1124. doi:10.1517/17425255.2010.486401

12. Tang LJ, Gu CL, Zhang P. Intraocular lymphoma. Int J Ophthalmol. 2017;10:1301-1307.

13. Zhuang L, Lai J, Chen K, et al. Intraocular involvement is associated with a high risk of disease relapse in primary central nervous system lymphoma. Oncol Rep. 2019;41:397-404.

14. Kim H, Jeong H, Yamaguchi M, et al. Prediction and prevention of central nervous system relapse in patients with extranodal natural killer/ T-cell lymphoma. Blood. 2020;136(22):2548-2556. doi:10.1182/ blood.2020005026
Cancer Management and Research

\section{Publish your work in this journal}

Cancer Management and Research is an international, peer-reviewed open access journal focusing on cancer research and the optimal use of preventative and integrated treatment interventions to achieve improved outcomes, enhanced survival and quality of life for the cancer patient.
The manuscript management system is completely online and includes a very quick and fair peer-review system, which is all easy to use. Visit http://www.dovepress.com/testimonials.php to read real quotes from published authors. 